Recepción: 26/ 06/ 2017

Aceptación: 28 / 07/ 2017

Publicación: 15/ 11/2017

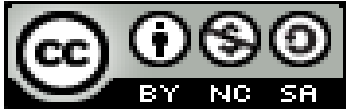

Ciencia de la educación

\title{
Contención emocional: una necesidad para la desactivación emocional Ante Stress-Burnout
}

Emotional restraint: a need for emotional deactivation Before Stress-Burnout

Restrição emocional: uma necessidade de desativação emocional antes do StressBurnout

Juan R. Morán-Quiñonez ${ }^{\text {I }}$ ramon.moran@uleam.edu.ec

Correspondencia: ramon.moran@uleam.edu.ec

I Magister en Docencia Mención Gestión en Desarrollo del Currículo, Especialista en Diseño Curricular por Competencias, Doctor en Psicología Clínica, Docente Universidad Laica Eloy Alfaro de Manabí, Manta, Ecuador. 


\title{
Resumen
}

Las emociones, los afectos y las pasiones son los elementos consustanciales que determinan la afectividad. Aunque en cualquier momento todos las experimentamos, no siempre somos conscientes de ello, especialmente cuando no las gestionamos adecuadamente, es decir cuando no tomamos en cuenta cuánto pueden hacernos daño o hacemos daño a los demás. Estas emociones son transitorias pero muchas veces muy intensas y pueden llegar a convertirse en dominantes por la forma que estas se expresan en el ser humano. Ellas pueden generar bienestar, satisfacción, placer, alegría, felicidad o incrustarse en el otro extremo con dolor, tristeza, ira, temor, enojo, desolación, rencor, resentimientos, estrés, burnout, depresión, que desencadenan alteraciones comportamentales, actitudinales, conductas violentas, desórdenes alimenticios, adicciones, llegando hasta acentuar o desencadenar enfermedades físicas, neurológicas (ictus-acv), cardiacas que pueden terminar hasta con la propia existencia. Sí no se logra esa capacidad de gestionarlas, al acumularse, es necesario aplicar técnicas que le posibiliten la evacuación. Una de estas es la desactivación emocional.

Palabras clave: gestión de las emociones; analfabetismo emocional; emociones tóxicas; simbolismo de las enfermedades.

\begin{abstract}
The emotions, the affections and the passions are the consubstantial elements that determine the affectivity. Although at any time we all experience them, we are not always aware of this, especially when we do not manage them properly, that is, when we do not take into account how much they can harm us or harm others. These emotions are transient but often very intense and can become dominant by the way they are expressed in the human being. They can generate wellbeing, satisfaction, pleasure, joy, happiness or embed in the other extreme with pain, sadness, anger, fear, anger, desolation, resentment, resentment, stress, burnout, depression, which trigger behavioral, attitudinal alterations, violent behaviors, eating disorders, addictions, even accentuating or triggering physical, neurological (stroke-acv), cardiac diseases that can end up with one's existence. If this ability to manage them is not achieved, when accumulating, it is necessary to apply techniques that enable evacuation. One of these is emotional deactivation.
\end{abstract}


Juan R. Morán Quiñonez

Keywords: emotion management; emotional illiteracy; toxic emotions; symbolism of diseases. 


\section{Resumo}

As emoções, as afeições e as paixões são os elementos consubstanciales que determinam a afetividade. Embora, em qualquer momento, todos os experimentemos, nem sempre estamos conscientes disso, especialmente quando não os gerenciamos corretamente, ou seja, quando não levamos em consideração o quanto eles podem nos prejudicar ou prejudicar os outros. Essas emoções são transitórias, mas muitas vezes muito intensas e podem se tornar dominantes pela forma como elas são expressas no ser humano. Eles podem gerar bem-estar, satisfação, prazer, alegria, felicidade ou incorporado no outro extremo com a dor, tristeza, raiva, medo, raiva, desespero, amargura, ressentimento, stress, esgotamento, depressão, provocando mudanças de comportamento, atitudes, comportamento violento , distúrbios alimentares, vícios, mesmo acentuando ou desencadeando doenças físicas, neurológicas (AVC), doenças cardíacas que podem acabar com a própria existência. Se essa capacidade de gerenciá-los não for alcançada, ao acumular-se, é necessário aplicar técnicas que permitam a evacuação. Uma delas é a desativação emocional.

Palavras chave: gerenciamento de emoção; analfabetismo emocional; emoções tóxicas; simbolismo de doenças.

\section{Introducción}

Las emociones, los afectos y las pasiones son los elementos consustanciales que determinan la afectividad. Aunque en cualquier momento todos las experimentamos, no siempre somos conscientes de ello, especialmente cuando no las gestionamos adecuadamente, es decir cuando no tomamos en cuenta cuánto pueden hacernos daño o hacemos daño a los demás.

Estas emociones son transitorias pero muchas veces muy intensas y pueden llegar a convertirse en dominantes por la forma que estas se expresan en el ser humano. Ellas pueden generar bienestar, satisfacción, placer, alegría, felicidad o incrustarse en el otro extremo con dolor, tristeza, ira, temor, enojo, desolación, rencor, resentimientos, estrés, burnout, depresión, que desencadenan alteraciones comportamentales, actitudinales, conductas violentas, desórdenes alimenticios, adicciones, llegando hasta acentuar o desencadenar enfermedades físicas, neurológicas (ictus-acv), cardiacas que pueden terminar hasta con la propia existencia. 
Si no se logra esa capacidad de gestionarlas, al acumularse, es necesario aplicar técnicas que le posibiliten la evacuación. Una de estas es la desactivación emocional, es un proceso intenso, consistente en aprender a cuestionar nuestras emociones y conjunto de creencias sobre nosotros mismos y sobre la vida, ante todo aquellas que nos bloquean y reprimimos, con el fin de abandonarlas y en última instancia, sustituirlas por otros pensamientos más constructivos.

Este trabajo tiene como objetivo: valorar las emociones en la afectación de la salud psicológica.

\section{Metodología}

Para cumplimentar el objetivo de este trabajo primeramente se presenta un análisis documental a partir de la revisión de la literatura publicada y en segundo lugar se muestra un estudio cualicuantitativo realizado en el mes de julio del año 2017, en diferentes sectores de la ciudad de Manta. Se utiliza una muestra de 735 adultos, de ambos sexos, de los cuales 349 fueron hombres y representa el $49 \%$ y 386 mujeres correspondiente al 53\%.

El rango de edad de la población fue de 12 a 70 años de edad: de 12 a 14 años corresponde un $10 \%$, de 15 a 20 un $50 \%$, de 21 a 25 un $25 \%$, de 26 a 30 un $3 \%$, de 31 a 40 un $4 \%$, de 41 a 70 años un $1 \%$.

Se utiliza una encuesta, diseñada y aplicada en años anteriores, con dos preguntas, que contenían siete y diez opciones de respuesta respectivamente. La primera inquiría: ¿cuál ha sido la causa de una crisis que usted ha tenido? y la segunda: ¿cuál fue su reacción ante ella?

\section{Desarrollo}

Un estudio publicado en el Current Biology (Jack, Garrod, \& Schyns, 2014) y llevada a cabo por investigadores del Instituto de Neurociencias y Psicología de la Glasgow University, en el Reino Unido, expresa que ha cambiado el paradigma respecto a cuáles son las emociones básicas del ser humano. El estudio concluye que no son seis las emociones base, sino solamente cuatro: enfado, miedo, alegría y tristeza. 
Las emociones positivas como el placer o la alegría suelen desaparecer o disminuir a lo largo del tiempo, mientras que las emociones negativas como la tristeza son de mayor duración. En concreto, estas últimas duran hasta 4 veces más que la alegría.

El punto de partida de las emociones que se expresan radican en el sistema límbico del cerebro, en el que intervienen varios mecanismos: fisiológicos, que son procesos involuntarios, por ejemplo aumenta el tono muscular, la respiración, se producen cambios hormonales, etc.; cognitivos, donde la información se procesa, tanto a nivel consciente como inconsciente e influye en nuestra experiencia subjetiva; conductuales, los cuales se expresan a través del movimiento del cuerpo, del tono de voz, de la cara, entre otros.

Se puede decir que el sentimiento es lo subjetivo de la emoción. Es decir, el sentimiento sería la combinación de la emoción instintiva y breve, junto con el pensamiento que obtenemos de forma racional de esa emoción.

Las emociones son un componente inmanente de nosotros, en cualquier cultura y edad, en determinadas circunstancias, pese a ser necesarias y adaptativas, nos ocasionan dolor, sufrimiento, malestar. ¿Pero, porque se produce ese sufrimiento? La respuesta radica en los engramas que se van estableciendo por el aprendizaje de cómo se gestionan esas emociones, es decir si las respuestas que aprendemos, son negativas, esas experiencias se acumulan y determinan una forma de pensar, una forma de reaccionar emocionalmente y luego, responder conductualmente, por tanto la clave radica no, en intentar luchar contra ellas, sino entenderlas, conocerlas, afrontarlas y enfrentarlas, saber que están ahí, pero aceptarlas como parte de nuestra existencia y nuestra experiencia, a sabiendas de que muchas veces no son como nos gustaría que fuesen.

La respuesta a un sentimiento puede ser física y/o mental, y está mediada por neurotransmisores como la dopamina, noradrenalina y la serotonina. Así mismo, los sentimientos duran más que la emoción, pues su duración proporcional al tiempo en que pensamos en ellos o como hayamos aprendido a gestionarlos. 
Cuando se está en fase de lucha-huida y la amígdala ha tomado el mando de los actos, normalmente ya es demasiado tarde. Por eso hay que aprender actuar antes. Es necesario acostumbrarse a detectar aquellas señales que indican que se va al camino de no poder dominar las emociones. Esta es la única forma de detener el proceso (o retrasarlo) antes de que sea demasiado tarde. Una vez que las emociones dominan, las reacciones emocionales como ansiedad, angustia, estrés, depresión, entre otras, se hacen presente, afectándonos de diversas maneras.

Seligman y Overmaier fueron de los primeros investigadores que se plantearon la cuestión sobre por qué un animal o una persona que sufría en sus propias carnes condiciones adversas y dolorosas constantes no hacía nada para abandonar dicha situación. Este hallazgo fue reportado en investigaciones con perros, y fueron seguidos posteriormente por algunos investigadores como Watson y Ramey, que estudiaron la indefensión aprendida en seres humanos. Por otra parte, no hay una situación en específico que genere la indefensión, es decir, muchas personas pueden vivir la misma situación adversa (incluso de manera grupal) y sin embargo reaccionar diferente ante ella. Fue Bernard Weiner quien consideró la influencia de la interpretación y la percepción que cada individuo tiene del evento en el desarrollo de la indefensión y también en la forma de afrontarla. (Ojeda, s/f).

Cuando alguien cae en la indefensión, lo manifiesta en tres déficits: el motivacional, el emocional y cognitivo. Una persona que empieza o que ya sufre de ella comienza a mostrar un retraso en la iniciación de respuesta voluntaria hasta que poco a poco deja de haberlas (déficit motivacional). De igual forma, comienzan a existir una serie de desórdenes conductuales, siendo los más habituales los estados de ansiedad y la depresión (déficit emocional), que van haciendo mella hasta el punto de que el afectado es incapaz de ver soluciones al problema que le atormenta (déficit cognitivo).

La respuesta a la pregunta de por qué no hace nada esa persona radica justamente en la afectación integral no solo de estos tres ámbitos (motivacional, emocional y cognitivo) sino también a nivel fisiológico. En consecuencia, no bastará con tomar la decisión de romper con el ciclo negativo, sino que implica desaprender la forma en que se procesa la situación aversiva o dolorosa. 
Lo triste a considerar es la facilidad con la que se puede llegar a desarrollar la indefensión aprendida. Todos somos vulnerables de adoptar este tipo de esquemas de pensamiento, pues pocas veces existe una educación emocional para poder enfrentarnos a ello.

Pero no son estos los únicos escenarios en que se pueden generar patrones relacionales que pueden desembocar en indefensión aprendida. La hay en la escuela, en el trabajo, en los grupos de amigos. Los estilos comunicativos y relacionales que generan indefensión aprendida no necesariamente se traducen en violencia física sino de tipo psicológico, económico, moral, entre otros.

En la literatura se habla de emociones tóxicas. Stamateac (2014) plantea que las emociones no pueden ser controladas desde fuera, sino que deben ser controladas desde dentro de la propia vida. Por tanto, de lo que se trata es aprender a identificar las emociones tóxicas y ser capaz de transformarlas en emociones positivas y sanas que conduzcan a una vida plena, armónica y feliz.

Dentro de las emociones tóxicas se habla de la ansiedad tóxica, angustia tóxica, insatisfacción crónica, apego (emocional) tóxico, enojo tóxico, envidia tóxica, miedo tóxico, vergüenza tóxica, depresión tóxica, frustración tóxica, duelo tóxico, llanto tóxico, culpa tóxica, rechazo tóxico, celos tóxicos.

Uno de los problemas que enfrenta el hombre contemporáneo es la falta de gestión de las emociones, lo cual implica que no se han desarrollado las habilidades necesarias para poder manejar, gestionar o lidiar con las respuestas emocionales que se generan como consecuencia de la interacción con el mundo, con las demás personas y con nosotros mismos en nuestro proceso permanente de readaptarnos a las condiciones que se imponen en el día a día.

Frente a cualquier respuesta que recibimos esta genera una emoción que puede ser positiva o negativa. La que nuestros neurotransmisores produzcan endorfinas y encefalinas, creándonos un ambiente de bienestar, placer y felicidad; mientras que la noradrenalina y serotonina son las que ocasionan la tristeza.

Mientras más sostenidos son las emociones negativas se pueden desarrollar un sinnúmero de afectaciones o alteraciones, que van desde las psicológicas, hasta las metabólicas como la 
diabetes, neurológicas (el ictus cerebral), musculares y articulaciones que se expresan en dolores, pues afectan directamente a las defensas (sistema inmunológico) del organismo.

"Es probable que una persona intente a través de conductas compulsivas y de adicciones, calmar su angustia, pero los resultados no van a ser los esperados". (La Bruna M. , 2010).

Las manifestaciones de labilidad emocional generan cambios de comportamientos en todas las áreas de la vida de una persona: puede repercutir de forma negativa en la vida familiar, laboral y social. Allí aparecerán las ansiedades, angustias, estrés depresiones, burnout, entre otros.

Según la OMS la depresión se define como el más común de los trastornos mentales. Afecta alrededor de 360 millones de personas en todo el mundo. Con una prevalencia estimada de 15 al $25 \%$, siendo mayor en mujeres, Entre los síntomas se encuentran, humor deprimido, pérdida de energía, trastornos del sueño, ansiedad, trastornos del apetito, trastornos sexuales, síntomas cognitivos y quejas somáticas.

En la cátedra de psicoterapia de intervención en crisis, bajo la dirección del responsable de la cátedra, durante los años 2015, 2016 y 2017 se indaga sobre las consecuencias que experimentan las personas ante una situación crítica, destacándose los componentes emocionales directos con un $62 \%$ (depresión, pánico, shock, agresivo, angustia), sin contar con las otras respuestas que son consecuencia de los estados emocionales. Ello ratifica lo dominante que son los estados emocionales negativos y cuanto afectan a las personas.

Los autores hablan de cuán necesario es aprender a escuchar a nuestro cuerpo, es decir, escuchar el lenguaje psicosomático. Con frecuencia la enfermedad expresa lo que realmente sentimos (emociones) con las cosas que nos pasan a nuestro alrededor.

El simbolismo de la enfermedad no es algo racional, cada uno puede darle una interpretación libre a las enfermedades que su cuerpo manifiesta, pero nunca debemos olvidarnos de ellas y si podemos, preguntémonos qué emociones pueden ocultarse tras ellas.

La anorexia y la bulimia son la expresión máxima de odio hacia uno mismo. Se trata de identificar qué es lo que vemos tan mal y cambiar la visión que tenemos de nosotros mismos para 

alcanzar la aceptación. La artritis se origina en una constante actitud de crítica hacia uno mismo o hacia los demás. Quienes padecen esta enfermedad suelen ser ellos mismos muy cuestionados, por lo cual frecuentemente son muy perfeccionistas. El asma denota un amor que sofoca. Así, afecta a personas que no sienten que tengan derecho a respirar por sí mismos. Los abscesos, quemaduras, cortes, fiebres, llagas e inflamaciones indican cólera que se expresa en el cuerpo.

Hay que buscar la manera de dejar salir la presión acumulada de una manera inofensiva. El cáncer es una enfermedad causada por un profundo resentimiento contenido durante mucho tiempo. Cuando en la infancia algo destruye nuestro sentimiento de confianza, nunca se olvida esa experiencia. Esto hace que el individuo viva compadeciéndose de sí mismo. La vida se presenta entonces como una serie de decepciones, con lo cual se vuelve muy sencillo culpar a otros de nuestros problemas. Las personas que tienen cáncer suelen ser también autocríticas.

Respecto a esto, el libro de David Ponce "Más amor y menos ibuprofeno". El título lo dice todo y va muy en la línea del poder que las emociones tienen en nuestro cuerpo.

Se necesita encontrar o identificar qué problemas emocionales están causando los dolores y los problemas físicos. Hay que aprender a entrenar y gestionar de manera adecuada las emociones, hay que conocerse profundamente y conectarse con la verdadera identidad y esencia.

Como dice William Osler, uno de los padres de la medicina moderna: "Es mucho más importante conocer qué tipo de paciente tiene la enfermedad que conocer el tipo de enfermedad que tiene el paciente".

Los resultados del instrumento aplicado demuestran que la mayor cantidad de fuentes generadoras de conflictos radica con padres, parejas, trabajo y con familiares, lo cual representa un acumulado del 78\% lo cual deja entrever la falta de habilidades sociales y emocionales que es lo que finalmente le lleva a conflictual su vida con consecuencias.

\begin{tabular}{|lcc|}
\hline REACCIÓN & CANTIDAD & \% \\
\hline & & \\
\hline Se deprimió & 313 & 43 \\
\hline Intentó suicidarse & 18 & 2 \\
\hline
\end{tabular}




\begin{tabular}{|lcc|}
\hline Entro en pánico & 32 & 4 \\
\hline Se dedicó a beber & 74 & 10 \\
\hline Se le quitó el apetito & 74 & 10 \\
\hline Entró en shock & 21 & 3 \\
\hline Se negó aceptar el problema & 100 & 14 \\
\hline Se volvió agresivo & 50 & 7 \\
\hline Se le presentó una angustia severa & 38 & 5 \\
\hline Otros & 15 & 2 \\
\hline & & \\
\hline Total & 735 & 100 \\
\hline
\end{tabular}

Tabla 1. Reacciones

Elaboración propia

En estos resultados las conductas de respuesta que emiten las personas frente a conflictos corresponden a las emocionales en su dominancia, pues reflejan un $62 \%$ entre depresiones, pánico, shock emocional, agresiones y angustia. Aunque también hay otros tipos de respuestas, son consecuencia de la afectación emocional ante la cual la mayoría de las personas reacciona de esta manera, lo cual es señal de que somatizan algunos de sus problemas. (Tabla1).

Al considerar que el ser humano es un ser biopsicosocial las respuestas a las crisis dependen en gran medida de la resiliencia, de la personalidad y de las capacidades de afrontamiento que las personas tengan.

Frente a las respuestas emocionales, se requiere siempre alternativas que posibiliten la desactivación emocional. Pero ¿cómo se logran estas? pues con la neuropsicoeducación, la reeducación, las psicoterapias y con los cambios en los estilos de vida que permitan un mejoramiento en esa calidad.

La educación es un componente esencial en la gestión de las emociones pues ello nos ayuda a prevenir los desajustes, no obstante, está demostrado que la neuropsicoeducación es la quien realmente genera cambios auténticos en las personas, porque para que un cambio se produzca se 
deben crear nuevas sendas neuronales con lo cual habrá un cambio actitudinal y/o comportamental. Eso sí es duradero.

Por otro lado, cuando estos cambios se complican es necesario acudir a los expertos, en estos casos a los psicólogos que hagan psicoterapia, quienes aplicando alguna de dichas técnicas conducirán a recobrar el control, la armonía en nuestras vidas, así hay técnicas como: relajación, mindfulness, meditación. Resiliencia, afrontamiento del estrés, EFT-Tapping (técnica de liberación emocional), Escritura expresiva. Aprender a fluir, Debriefing o CISD (CriticalIncident stress Debriefing), Defusing o Desactivación. Brainspotting, que cumplen estos objetivos.

Hay otras estrategias que pueden adoptarse para el control emocional, y tienen que ver con acciones de la vida común, por ejemplo:
a. Entiende tus emociones.
b. Práctica la escucha activa.
c. Haz ejercicio físico.
d. No te tomes mal las críticas.
e. Trabaja la autoaceptación.
f. Adopta una actitud optimista.
g. Piensa en lo bueno y no en lo malo.
h. Retírate en el momento adecuado.
i. No reacciones de golpe.
j. Apúntate a un curso de inteligencia emocional.
k. Practica deportes.
1. Aliméntese adecuadamente.

\section{Conclusiones}

- Los conflictos con los padres son las crisis que más se muestran dentro de la población debido a la diversidad de problemas presentados en el hogar, siendo la reacción o respuesta ante aquello deprimirse por la situación dada, debido a que la adolescencia es una etapa crucial de cambios físicos, psicológicos y emocionales, por lo que es común 
que existan en el hogar conflictos con los padres que puedan provocar este tipo de reacción.

- Las crisis son cotidianas en la sociedad y se manifiesta en cualquier tipología familiar y nivel económico, sin embargo, las reacciones que se presentan van a depender de los factores de protección de los individuos, ya sea psicológico, social, emocional, incluso biológico ante una crisis.

- Ante la falta de la habilidad relacionada con gestionabilidad de las emociones, las consecuencias son de carácter catastrófica en ciertos momentos, al somatizar las respuestas emocionales.

\section{Referencias Bibliográficas}

Jack, R., Garrod, O., \& Schyns, P. (2014). Dynamic Facial Expressions of Emotion Transmit an Evolving Hierarchy of Signals over Time. Current Biology

La Bruna, M. (2010). Cómo las emociones afectan la salud del corazón. Recuperado el febrero de 2015, de http://www.diariojunin.com/notasuplemento/2037_como-las-emociones-afectan-lasalud-del-corazon.html

Ojeda, C. (s/f). La indefensión aprendida: ahondando en la psicología de la víctima. Recuperado el febrero de 2016, de https://psicologiaymente.net/psicologia/indefension-aprendida

Osler, W. Citado por: Rodrigo Sáez L, Linares Rodríguez A. Síndrome de colon irritable. Oviedo: Duphar Farmacéutica, 1992

Ponce, D. (2012) HYPERLINK "http://www.masamorymenosibuprofeno.com/" \t "_blank" Más amor y menos ibuprofeno

Stamateac, B. (2014) Emociones tóxicas 